\title{
Illustrated Review of the Embryology and Development of the Facial Region, Part 2: Late Development of the Fetal Face and Changes in the Face from the Newborn to Adulthood
}

P.M. Som and T.P. Naidich

\author{
ABSTRACT
}

SUMMARY: The later embryogenesis of the fetal face and the alteration in the facial structure from birth to adulthood have been reviewed. Part 3 of the review will address the molecular mechanisms that are responsible for the changes described in parts 1 and 2.

P art 1 of this 3-part review primarily dealt with the early embryologic development of the face and nasal cavity. Part 2 will discuss the later embryonic and fetal development of the face, and changes in facial appearance from neonate to adulthood will be reviewed.

\section{Formation of the Palate}

Between the sixth and 12th weeks, the palate is formed from 3 primordia: a midline median palatine process and paired lateral palatine processes (Fig 1). In the beginning of the sixth week, merging of the paired medial nasal processes forms the intermaxillary process. From this, a wedge-shaped primary anterior mesenchymal mass extends posteriorly, between the internal surfaces of the developing maxillae, to form the primary palate (Fig $1 B$ ). The primary palate then gives rise to the premaxilla, the anterior median portion of the maxilla that encloses the 4 upper incisors. The secondary palate is the primordium of the remaining hard and the soft palates. Later in the sixth week, paired lateral palatine processes arise as medial mesenchymal projections from each maxillary process (Figs 1 and 2). Initially these grow inferiorly, between the developing tongue and the developing alveolus (see the sections on the mandible and teeth) (Fig 2A). However, as the maxilla and mandible elongate, the tongue is pulled downward away from the lateral palatal processes. During the seventh and eighth weeks (Fig 2B), the palatal shelves elevate into a horizontal position above the tongue (Fig $2 C,-D$ ). This change in orientation is facilitated by the release of hyaluronic acid by the mesenchyme of each palatal process. When the palatal shelves

From the Department of Radiology, Mount Sinai School of Medicine, New York University, New York, New York.

Please address correspondence to Peter M. Som, MD, Department of Radiology, The Mount Sinai Hospital, One Gustave Levy Place, New York, NY 10029; e-mail: Peter.Som@MSSM.edu

- Indicates open access to non-subscribers at www.ajnr.org

http://dx.doi.org/10.3174/ajnr.A3414 first make contact, each is completely covered by a homogeneous epithelium. A special epithelium arises at the edge of each palatal shelf, facilitating the eventual fusion of these shelves. The epithelium on the nasal cavity surface of the palate will differentiate into columnar ciliated epithelium. The epithelium on the oral cavity side of the palate will differentiate into stratified squamous epithelium.

The 2 palatal shelves also fuse with the triangular primary palate anteromedially to form a y-shaped fusion line. The point of fusion of the secondary palatal shelves with the primary palate is marked in the adult by the incisive foramen. The fusion of the 2 palatal shelves also results in a lengthening of the nasal cavity and carries the posterior choana back toward the pharynx. Ossification gradually occurs in the primary palate and then extends into the palatal processes to form the hard palate. The posterior portions of the palatal processes do not ossify. Rather they extend posteriorly to the nasal septum and fuse to form the soft palate ad midline uvula. ${ }^{1,2}$

\section{Nasal Septum}

The nasal septum develops downward and posteriorly from the internally merged medial nasal processes and the frontonasal process. Fusion between the nasal septum and the palatal processes starts anteriorly during the ninth week and is completed by the 12th week (Fig 2). ${ }^{1,2}$

\section{Mandible}

During the fourth-to-fifth weeks, the mandibular processes gradually enlarge and merge in the midline. Between the fifth and eighth weeks, neural crest cells of the first pharyngeal arch give rise to left and right cartilaginous rods called the Meckel cartilages. These form the cores around which the membranous bone of the lower jaw develops. The mandibular processes form the lower lip, the lower jaw, and the lower cheek regions. The mentum marks the site where the 2 mandibular 


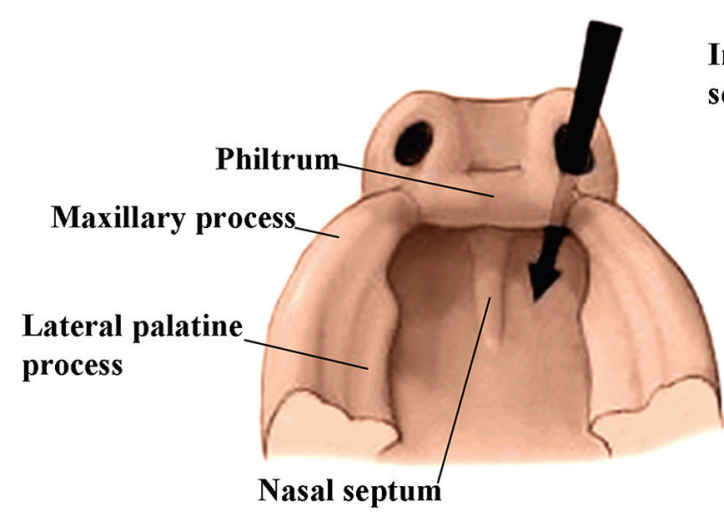

A

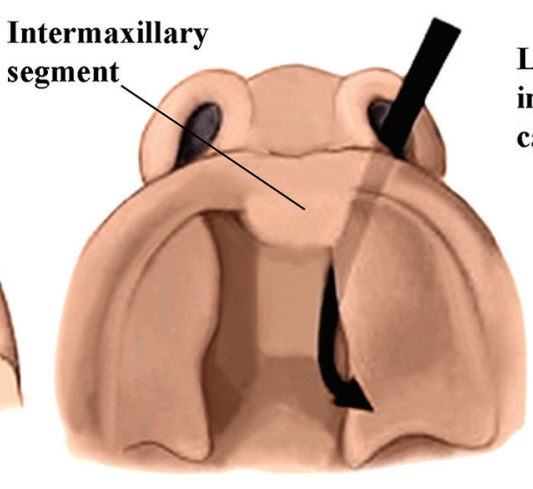

B

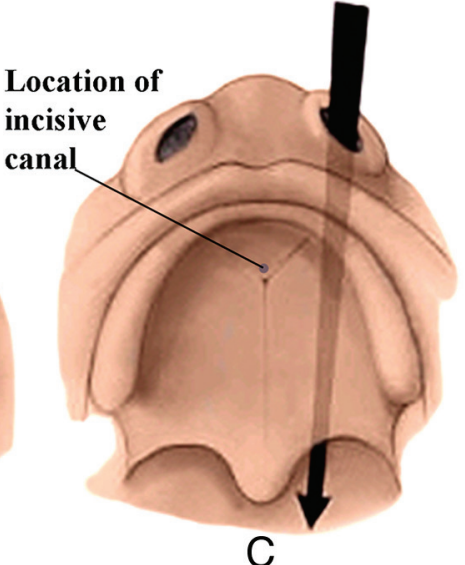

C

FIG 1. Drawings from below show the development of the palate from 6 to 7 weeks $(A), 7$ to 8 weeks $(B)$, and 8 to 10 weeks $(C)$. The lateral palatine processes grow medially and eventually merge in the midline and with the intermaxillary segment (primitive palate). The incisive canal marks the junction of the primitive and secondary palates. (Modified with permission from Levine HL, Clemente MP, eds. Chapter 1, Surgical Anatomy of the Paranasal Sinus. China: 2005. Sinus Surgery Endoscopic and Microscopic Approaches. Figures 1-3. Thieme Medical Publishers Inc., Georg Thieme Verlag Stuttgart).
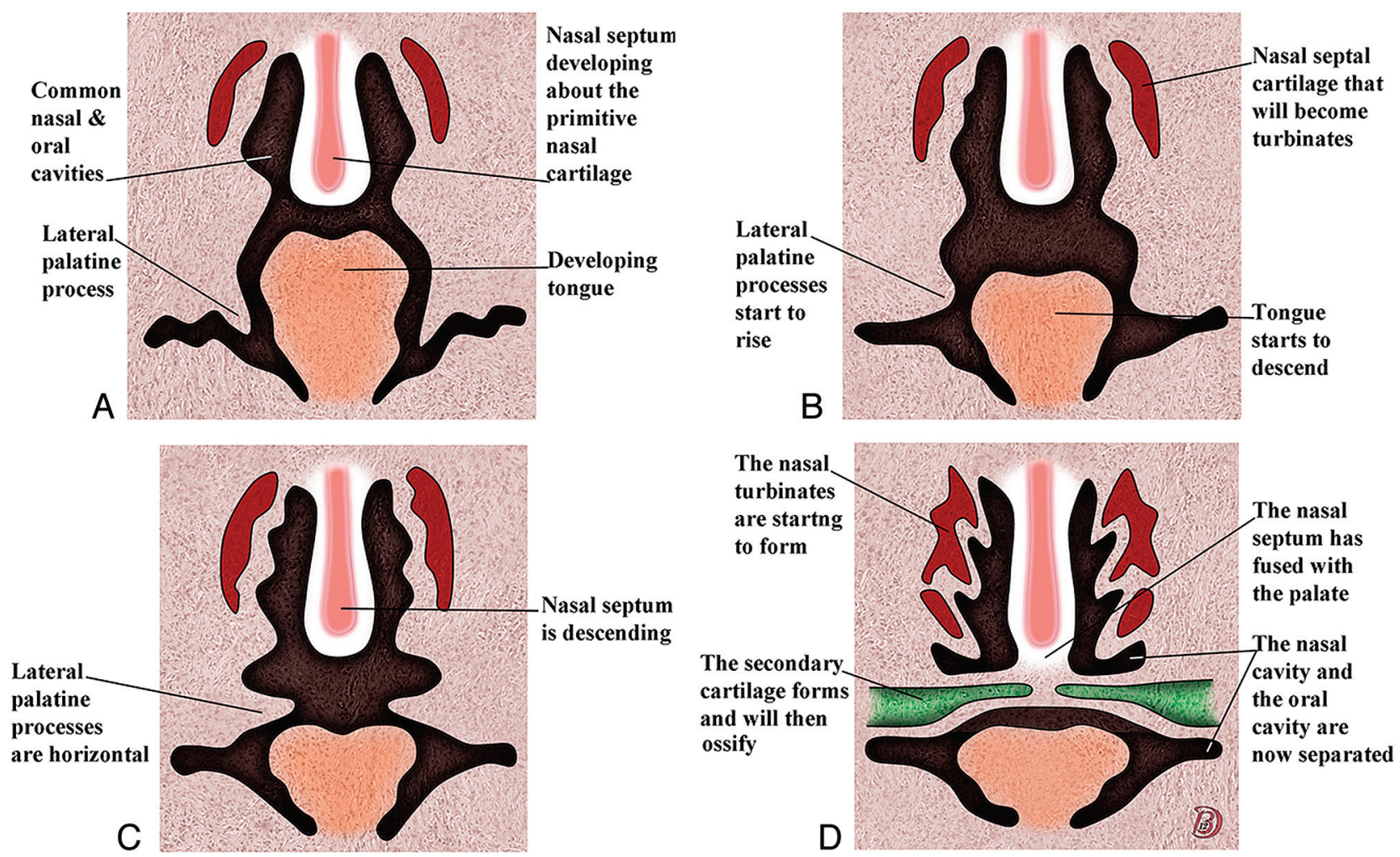

FIG 2. Serial frontal diagrams $(A-D)$ from approximately 6-10 fetal weeks shown just posterior to the intermaxillary segment illustrate the progressive development of the secondary palate and its fusion with the nasal septum.

processes merge in the midline. A partial or incomplete merger of these mandibular processes forms the common midline chin dimple or cleft.

\section{Gingiva, Lips, and Teeth}

Until the end of the sixth week, the primordial jaws comprise only masses of mesenchymal tissue with no differentiation of the lips and gingivae. At the end of the sixth week, a curvilinear thickening of ectoderm, the labiogingival lamina, grows into the underlying mesenchyme. Most of this lamina degenerates, creating a labiogingival groove or sulcus between the lips and gingivae (Fig 3A). A small midline remnant of the labiogingival lamina persists as the frenulum of the upper lip (Fig $3 B$ ).

Shortly after in the sixth week, a second lamina, the dental lamina, arises in the more buccal margin of the developing gingiva of both jaws. The dental lamina eventually will give rise to 10 spherical tooth buds that penetrate the mesenchyme of both jaws. The tooth buds first appear in the anterior mandible followed by the anterior maxilla. Budding then continues progressively posteriorly to form 10 deciduous teeth each in the maxilla and mandible. From about the 10th fetal month, deep 


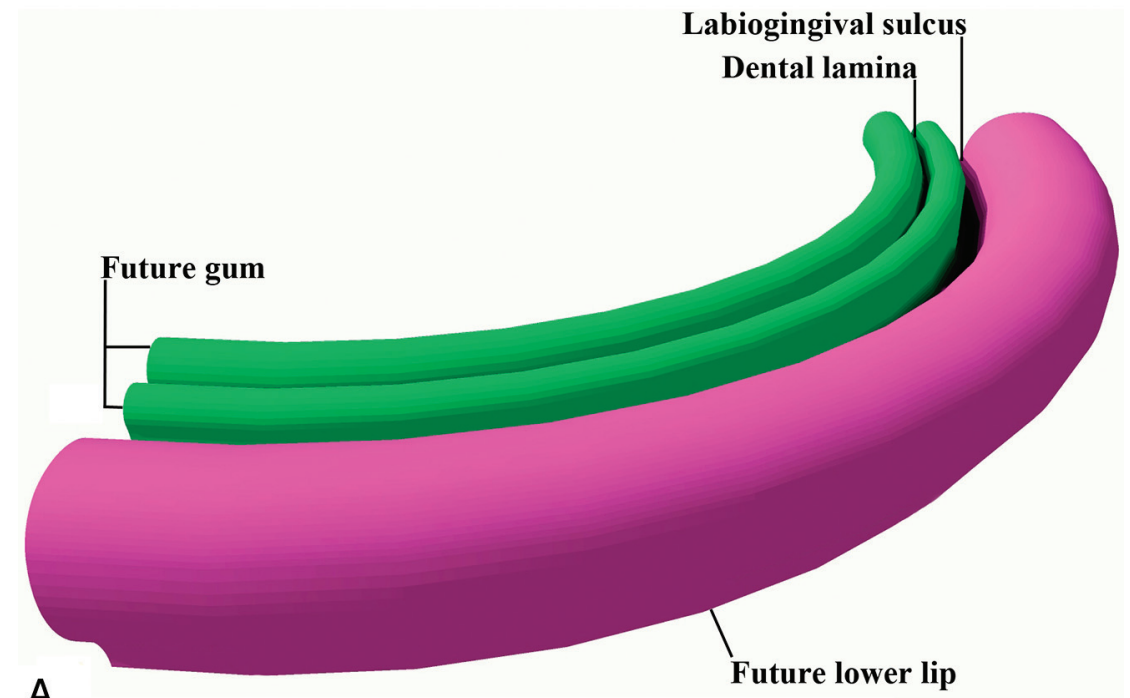

A

Future lower lip

B

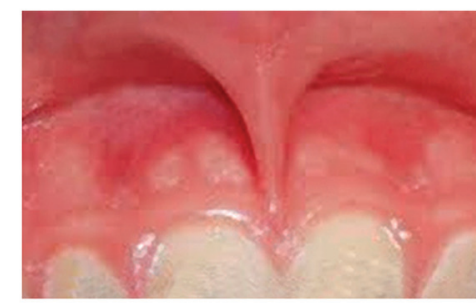

FIG 3. Drawing from above and in front $(A)$ of the developing lips and gums. The labiogingival lamina develops in the common mesenchymal tissue of this region. When it dissolves, the labiogingival sulcus that remains separates the lips and gums. The dental lamina develops just behind this region and will give rise to the dental buds, which will form the deciduous and permanent teeth. $B$, Frontal photograph shows the frenulum of the upper lip, the only remaining vestige of the labiogingival lamina.

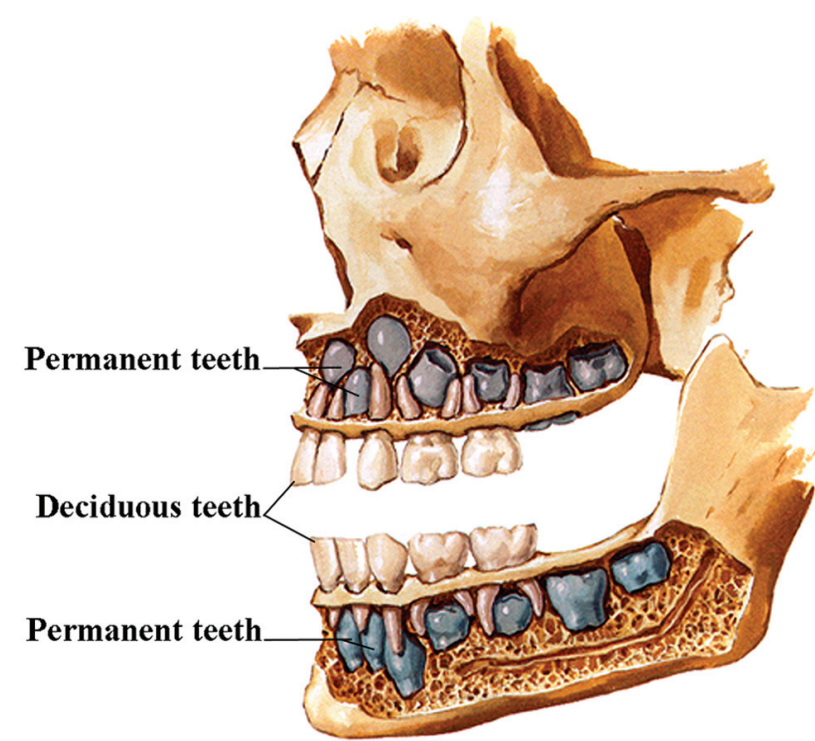

FIG 4. Lateral drawing of the developing teeth. Note that the permanent teeth (blue) develop medial to the deciduous teeth. (Modified with permission from Frank H. Netter, Atlas of Human Anatomy, 5th Edition, Saunders Elsevier, Philadelphia, 2011, Figure 56. Netter Illustrations from www.netterimages.com, oElsevier Inc, All rights reserved).

components of the dental lamina create the buds for the permanent teeth along the lingual aspects of the deciduous teeth (Fig 4). The permanent molar teeth have no deciduous precur- sors and arise directly from posterior extensions of the dental laminae.

By the 10th week, a mesenchymal condensation called the "dental papilla" invaginates into each tooth bud, resulting in the formation of a cup-shaped enamel organ (Fig 5A). The enamel organ has an inner epithelium, an outer epithelium, and a middle enamel reticulum (enamel pulp) (Fig $5 B,-E$ ). The dental papilla contains attenuated collections of stellate cells, which eventually give rise to most of the tooth proper, including the pulp cavity, the dentin, and the vasculature of the tooth. The enamel organ and dental papilla are surrounded by a mesenchymal concentration called the "dental sac." This sac will develop into the fibrous connective tissue (periodontal ligament) that attaches the roots of the teeth to the alveolar bone (Fig 5C). The inner enamel epithelium later differentiates into the ameloblasts that produce the tooth enamel. By the third month, the mesenchymal cells in the dental papilla adjacent to the inner enamel epithelium differentiate into odontoblasts. These produce predentin and deposit the predentin adjacent to the inner enamel epithelium (Fig $5 C$ ). In the sixth month, the predentin calcifies to become the dentin of the tooth.

The crown of the tooth refers to that part of the tooth covered by enamel and projecting above the gum line. The formation of the tooth root begins when the inner and outer enamel layers penetrate into the mesenchyme to form the epithelial root sheath (Hertwig epithelial root sheath) (Fig 5D). Root formation takes place after the crown formation has been completed. The root 

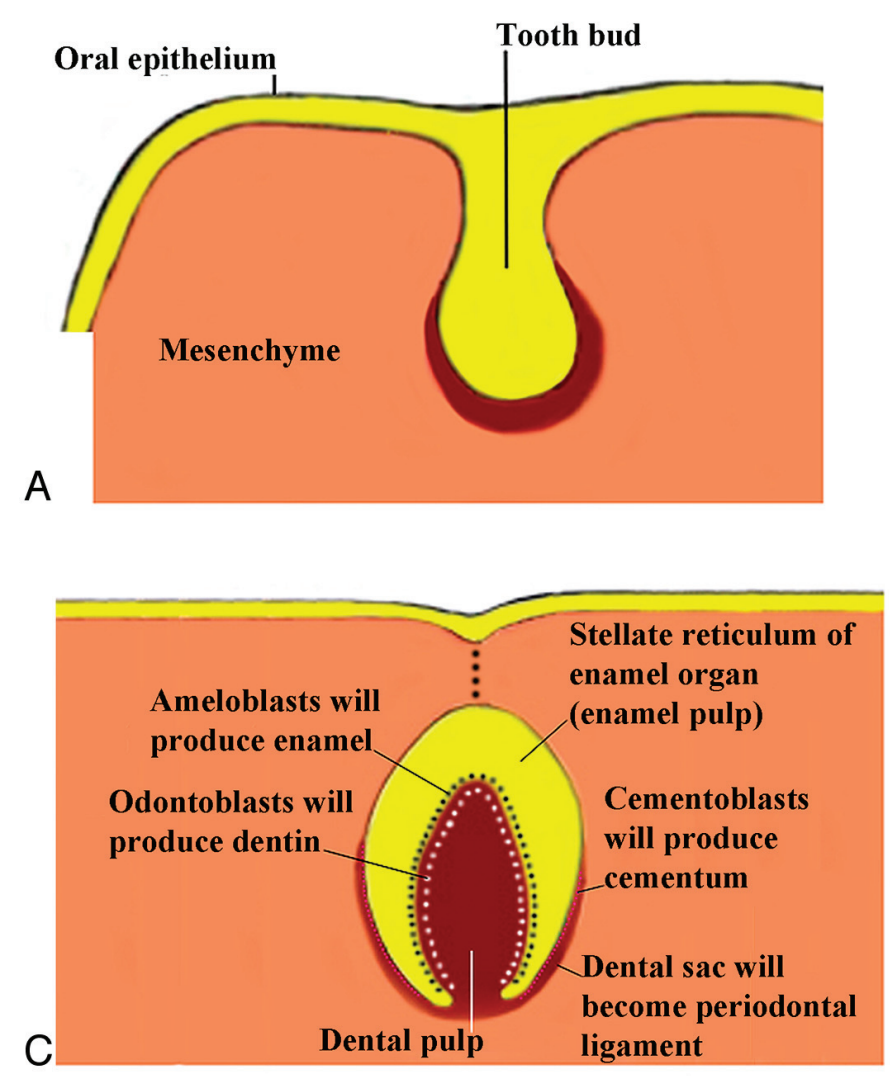
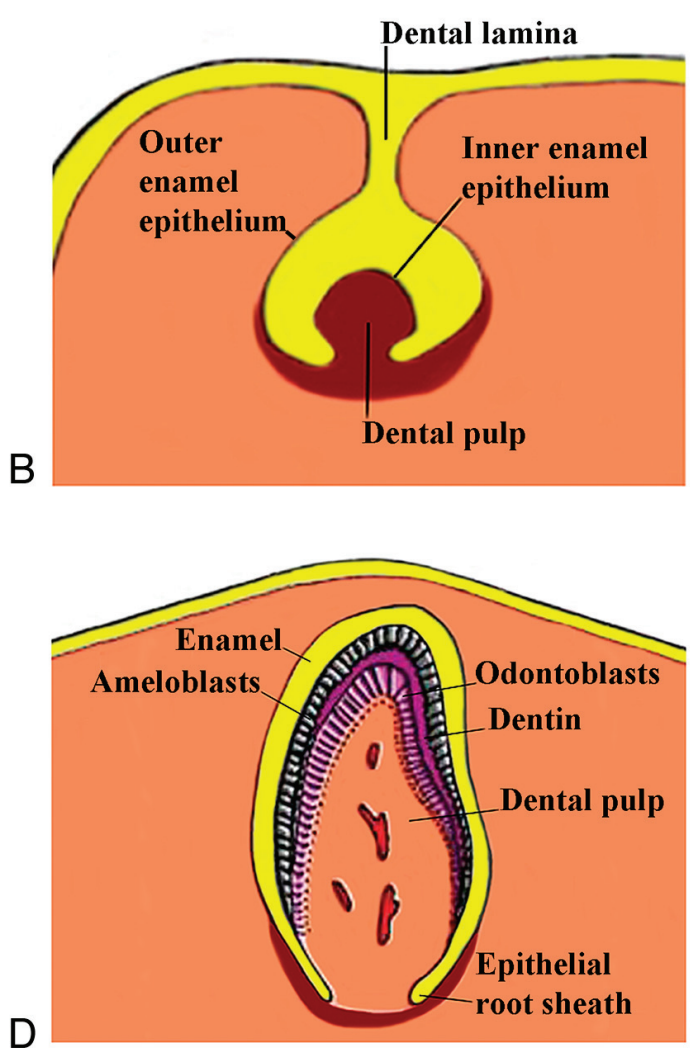

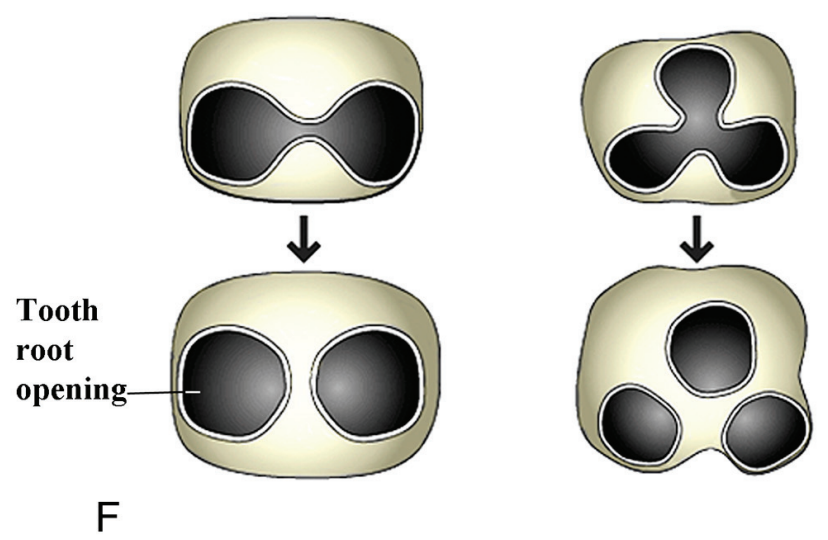

$E$

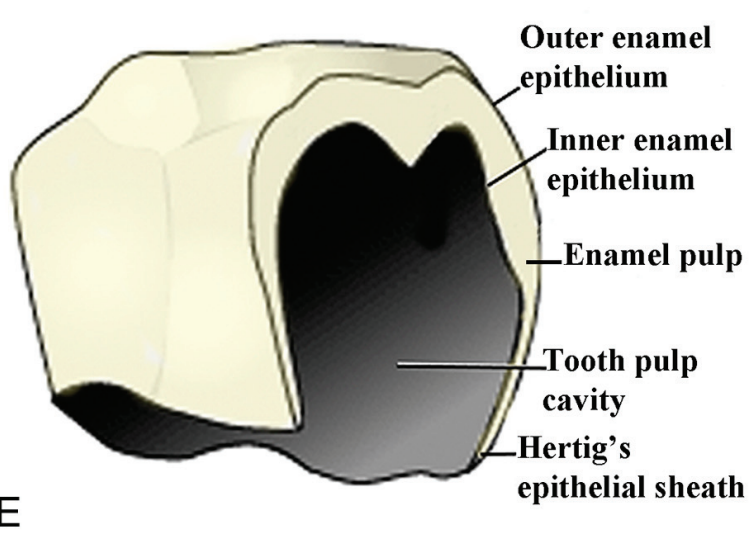

FIG 5. Drawings of the progressive development of the teeth from the tooth bud stage that comes from the dental lamina to the adult tooth. (Modified from http://embryology.ch/anglais/sdigestive/gesicht05.htm and www.embryo.chronolab.com/teeth.html).

canals arise through extension and later fusion of the enveloping root sheath (Fig 5F). The inner cells of the dental sac differentiate into cementoblasts, which will produce the cementum of the tooth. Increasing amounts of dentin reduce the pulp cavity to the narrow root canal through which the vessel and nerves pass. The deciduous teeth erupt through the gingiva from 6-24 months after birth. ${ }^{3}$

\section{Facial Muscles}

The facial muscles start to develop between the third and eighth weeks when the mesoderm of the second branchial arch starts to thicken just caudal to the first branchial groove. Between the sixth and eighth weeks, sheet-like collections of premyoblasts and early myoblasts extend from this attenuated mesenchyme to form 5 laminae on each side of the face, which extend into the superficial portions of the future temporal, occipital, cervical, and mandibular regions. On each side of the face, the infraorbital lamina and the occipital platysma are the first laminae to appear (Fig. 6). Each infraorbital lamina forms the zygomaticus major, the zygomaticus minor, the levator labii superioris, the levator labii superioris alaeque nasi, the superior part of orbicularis oris, the compressor naris, the dilator naris, the depressor septi, the orbicularis oculi, the frontal belly of occipitofrontalis, the corrugator supercilii, and the procerus muscles. Each occipital lamina forms the occipital 

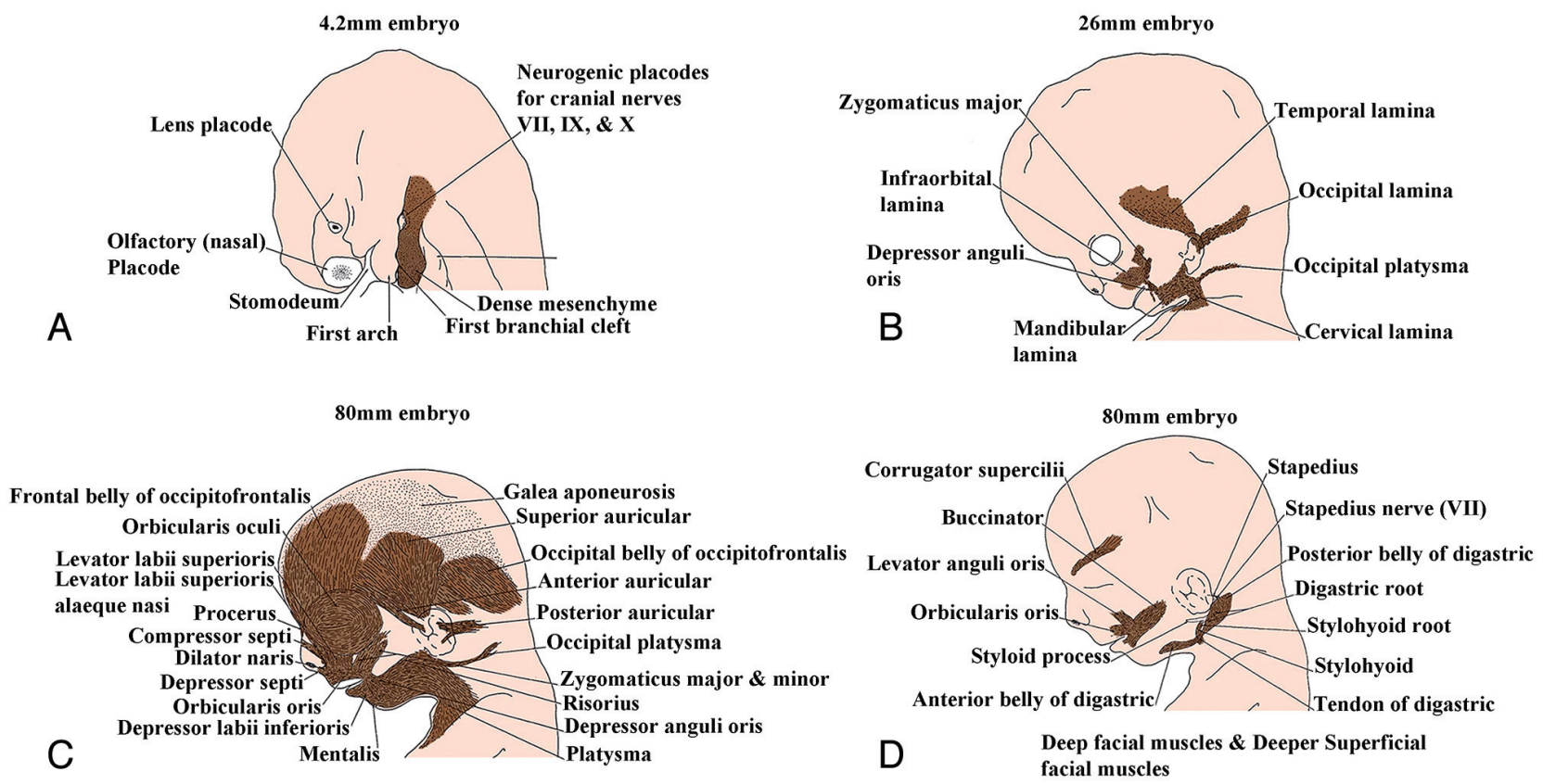

FIG 6. Sagittal drawings of the progressive development of the facial muscles from the dense mesenchyme that arises near the first branchial cleft. (Modified with permission from Gasser R. The Development of the Facial Muscles in Man. Am J Anat 1967;120:357-376).
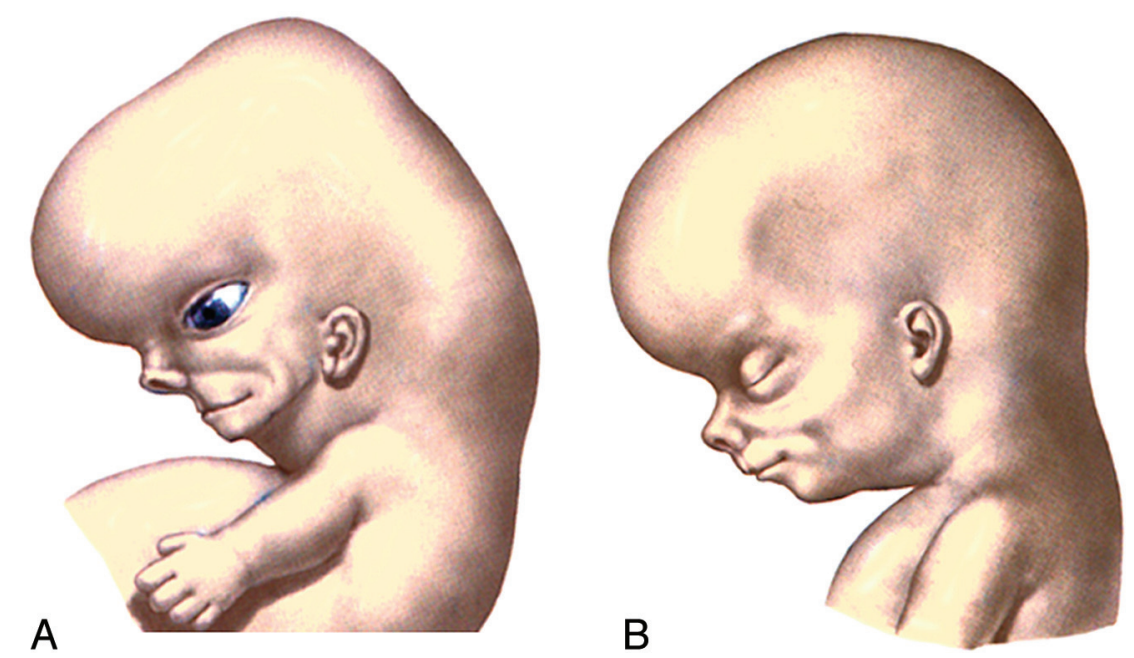

FIG 7. Lateral drawings of a 7- to 8-week embryo $(A)$ and an 8- to 10 -week fetus $(B)$ show that the opening of the external auditory canal remains stationary but appears to rise because the progressive elongation of the jaw creates this impression. (Modified with permission from Netter's Atlas of Human Embryology. Edited by Cochard, L.R., PhD. 2002. Icon Learning Systems, Teterboro, New Jersey, Figure 9.27. Netter Illustrations from www.netterimages.com, oElsevier Inc, All rights reserved).

belly of the occipitofrontalis muscle. Each temporal lamina develops into the superior auricular muscles. Each mandibular lamina forms the mandibular part of platysma, the depressor labii inferioris, the mentalis, the risorius, the depressor anguli oris, the buccinator, and the levator anguli oris muscles. Mesenchymal cells adjacent to the first branchial cleft form the anterior auricular muscle on each side of the face. The deep muscles form separately for the mesoderm, and these muscles comprise the posterior belly of digastric, the digastric tendon, the stapedius, and the stylohyoid muscle on each side of the face. $^{4}$

\section{Remodeling of the Face}

During the early fetal period, the nose is flat, the mandible appears small, and the orbits face laterally. These structures will grow into their adult configurations as facial development is completed. As the brain enlarges, the cranial vault expands to each side causing the orbits to face forward. The ostia of the external auditory canals remain stationary but appear to rise because the jaw elongates and grows downward (Fig 7). Early on, the stomodeum extends across nearly the full width of the embryonic face. By the seventh-toeighth weeks, differential growth of the facial elements brings the eyes and lateral portions of the maxilla and mandible to a more 


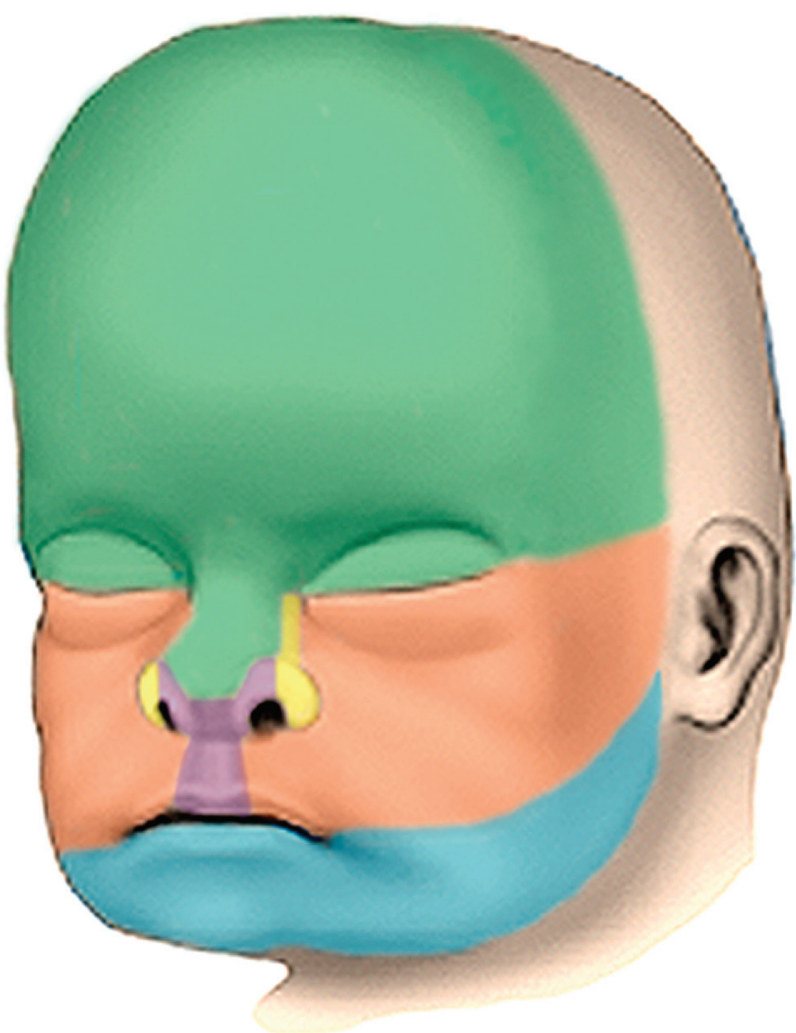

FIG 8. Drawing in an anterior oblique view of the late fetal face showing the contributions of the various facial processes. Green indicates the frontonasal process; yellow, the lateral nasal processes; purple, the medial nasal processes; orange, the maxillary processes; and blue, the mandibular processes.

frontal location. Although the expansion of the cranial vault does make the mouth appear smaller, it is the formation of the cheeks by progressive fusion of the maxillary with the mandibular processes at the lateral angles of the mouth that most reduces the size of the mouth. The coronal plane along which the oropharyngeal membrane originally attached eventually corresponds to the plane of the Waldeyer ring. The progressive depth of this ring in the postnatal period is due to the dominant differential growth of the ventral face.

\section{Summary of Contributions}

The frontonasal process forms the forehead, the bridge, and dorsum of the nose and the nasal bones. The medial nasal processes form the columella of the nose, the philtrum, the perpendicular plate of the ethmoid bone and the vomer, the cribriform plates, and the primary palate. The lateral nasal processes form the sides and alae of the nose. The maxillary processes form the upper cheek regions and most of the upper lip, the maxilla, zygoma, and secondary palate. The mandibular processes form the chin, lower lip, lower cheek regions, and the mandible (Fig 8).

\section{Pinna of the Ear}

At the beginning of the sixth week, 6 auricular mesenchymal hillocks appear, 3 on either side of the first branchial cleft or groove. During the seventh week, the pinna of the ear will have taken its adult shape (Fig 9).

\section{Eyelids}

In the sixth week, the eyelids start to form from neural crest mesenchyme and from 2 cutaneous folds of ectoderm that grow over the cornea. The eyelids grow rapidly until they meet and fuse to each other in the 10th week. At this time, a persistent epithelial lamina arises between the eyelids. Between the 26th and 28th weeks before the eyelids reopen, eye lashes and small meibomian glands begin to differentiate from the common epithelial lining.

\section{Later Facial Growth}

At birth, the skull has a sagittal suture system that divides the cranium and face into left and right halves. Anteriorly, this system
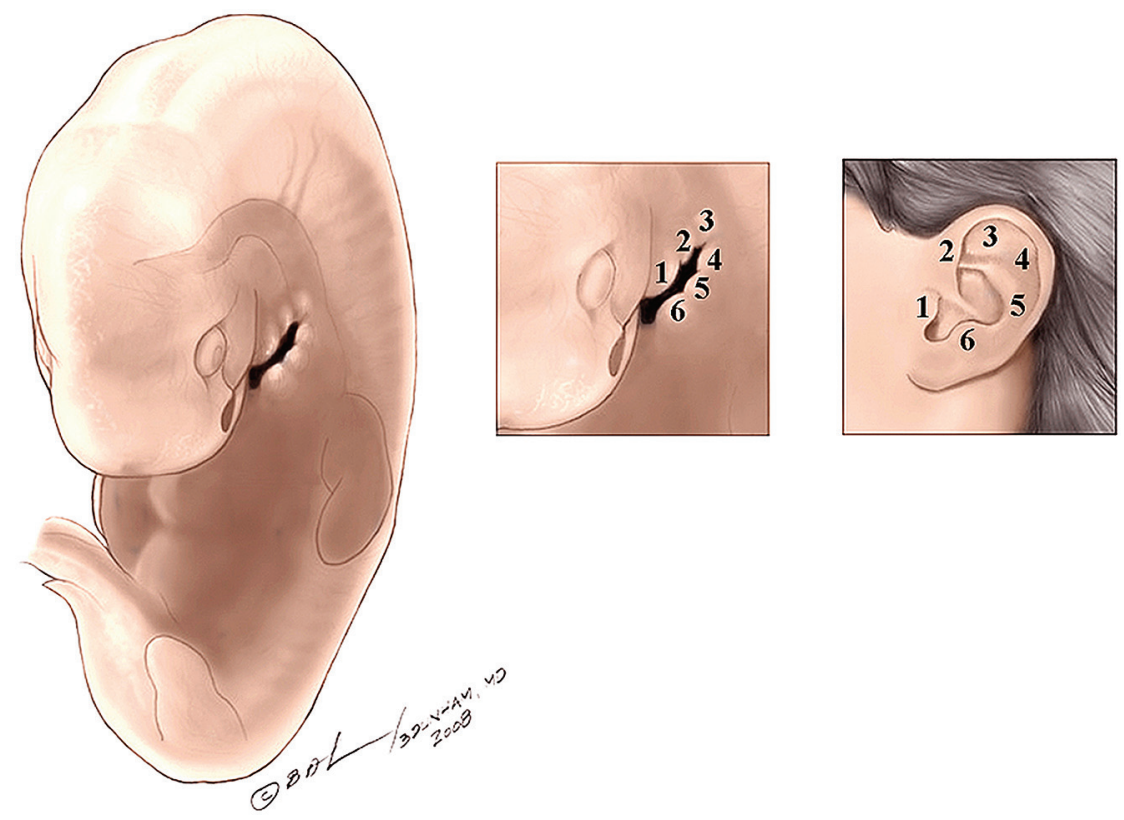

FIG 9. Lateral oblique drawings of the 6 hillocks that develop about the first branchial cleft and how they eventually form the pinna of the ear. 

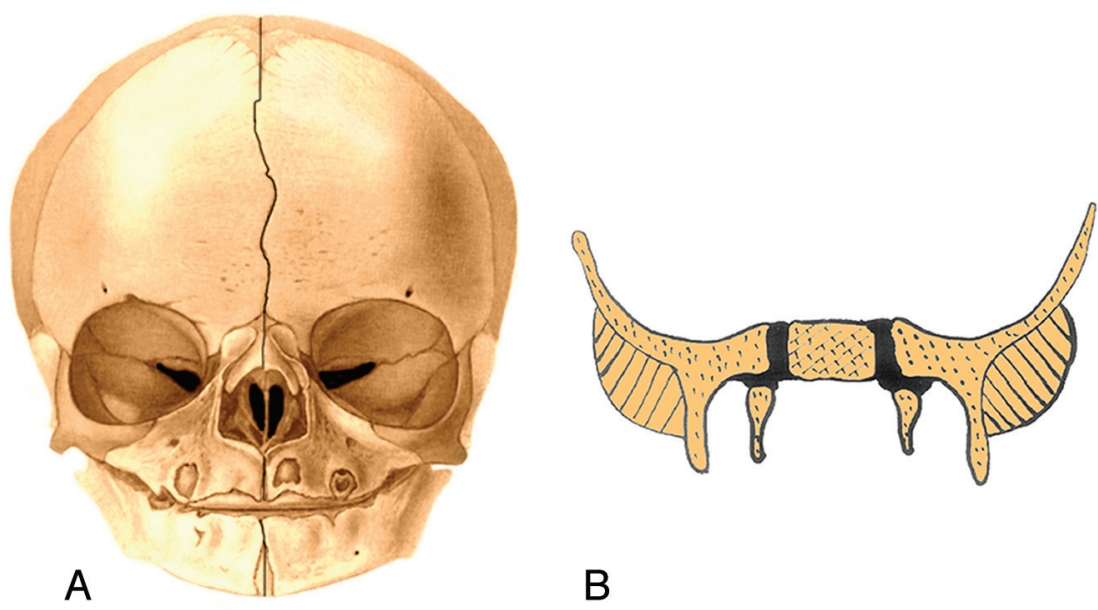

B

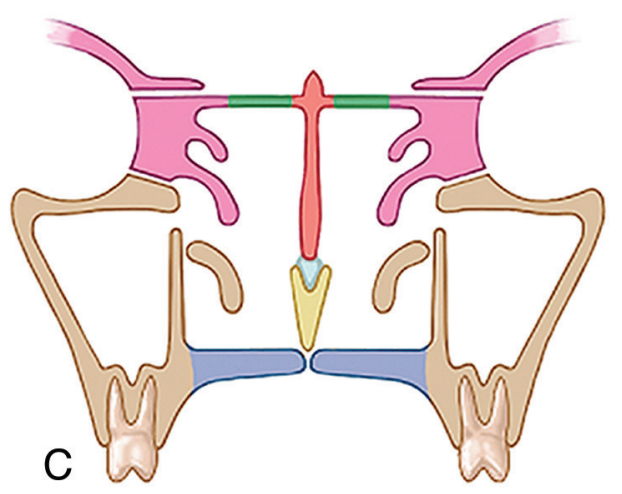

FIG 10. Frontal drawing of a neonate skull $(A)$ shows the sagittal suture system that divides the cranium and face into 2 halves. This system is made up of the metopic suture, the internasal suture, the intermaxillary suture, and the mandibular symphysis (outlined with a black line). (Modified with permission from Sobotta: Atlas der Anatomie des Menschen. @Elsevier GmbH, Urban \& Fischer Verlag Munchen. Volume 1, Edited by Putz, R. and Pabst, R. Lippincott Williams and Wilkins, Philadelphia 2001, Figure 82). B, Frontal drawing of the body of the sphenoid bone, the greater sphenoid wings, and the cartilage between them (black). The sagittal suture system divides to run on either side of the body of the sphenoid bone because it is separated from the greater sphenoid wings by cartilage. C, Frontal drawing of the midfacial structures at approximately 3 years of age. The union of the ethmoid bodies (pink) with the perpendicular plate (orange) as a result of ossification of the cribriform plates (green) makes the ethmoid bone a single bone and stabilizes the interocular and upper nasal regions. The maxilla is beige; vomer, yellow; septal cartilage, light blue.

is made up of the metopic suture, the internasal suture, the intermaxillary suture, and the mandibular symphysis (Fig $10 A$ ). Posteriorly, the sagittal suture system splits around the body of the sphenoid bone, along the cartilage between the body of the sphenoid and the greater wings of the sphenoid (Fig 10B). The sagittal system does not bisect the entire skull however because other midline structures extend from the foramen magnum to the nasion. These structures comprise the basioccipit, basisphenoid, lesser wings of the sphenoid, the perpendicular plate of the ethmoid, and the interorbital portion of the frontal bone. ${ }^{5}$

By $8-9$ weeks, the initial skeleton of the face is cartilaginous and composed of the nasal capsule in the upper face and Meckel cartilage in the lower face. The chondrocranium forms the skull base. By 12 weeks, most of the ossification centers have appeared in the membranous bones, and the enchondral ethmoid bone has started to ossify. Ossification then proceeds within these bones. During the late fetal period and until the first postnatal year, growth in the width of the craniofacial skeleton occurs at the midsagittal suture system. The main mechanism of this growth is the progressive enlargement of the brain and growth of the cartilage between the body and greater wings of the sphenoid bone.

In the first year of life, the metopic suture unites, and soon after, the mandibular symphysis unites. Then the greater sphenoid wings unite with the sphenoid body. These changes close the midline sagittal suture system, and it ceases to be a growth site. ${ }^{5}$

At approximately 3 years, ossification of the cribriform plates unites the ethmoid bodies with the perpendicular plate, creating a single ethmoid bone and stabilizing the interocular and upper nasal regions (Fig 10C). It has been suggested that the progressive growth of the nasal septal cartilage "pushes" the midface forward and thus contributes to the facial anteroposterior growth. ${ }^{5,6}$

After the third year, separation of the maxillary bones is still possible, as is separation of the zygomatic bone from the maxilla. However, with completion of the growth of the orbits between the seventh and 10th years, further outward movement of the maxilla and zygoma ceases at these sutures. Further growth of the upper facial skeleton takes place by surface bone deposition in association with internal bone resorption. ${ }^{5}$ 


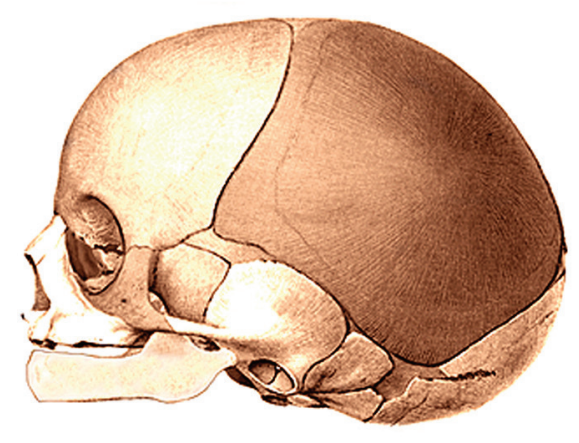

A

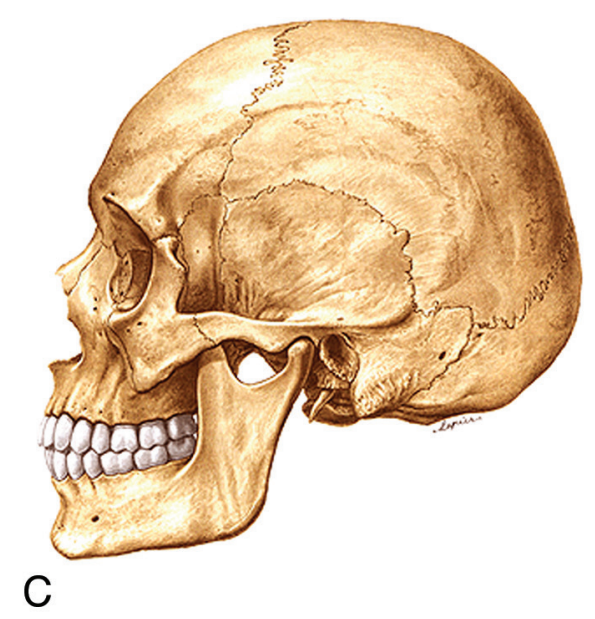

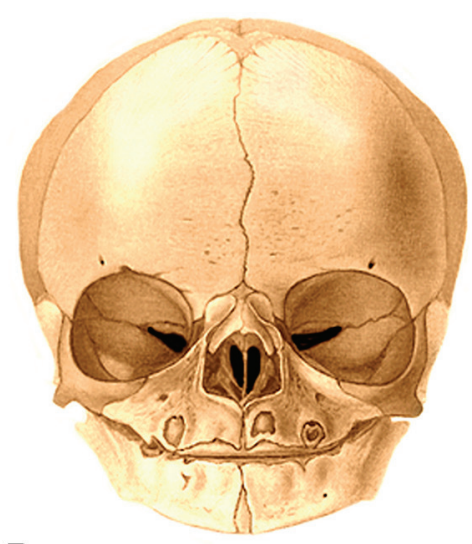

B

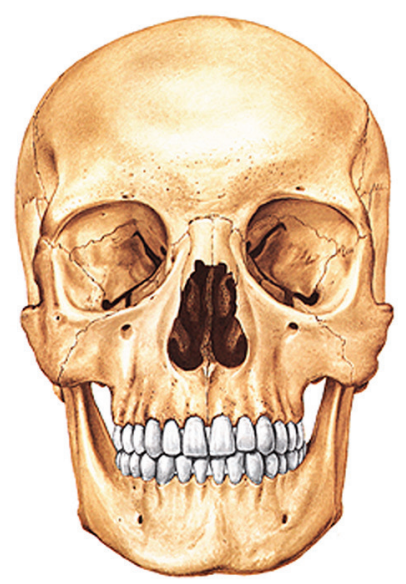

D

FIG 11. Lateral $(A)$ and frontal $(B)$ drawings of neonate facial bones and skull and adult facial bones and skull in the lateral $(C)$ and frontal $(D)$ views. In general, the facial structures grow proportionally more and for a longer time the further they are from the neurocranium. Thus, growth of the mandible begins later and continues longer than midfacial and orbital development. The forehead grows in an anterior and slightly upward direction. The forward and upward growth of the forehead contributes to elevation and widening of the nasal bridge. (Modified with permission from Sobotta: Atlas der Anatomie des Menschen. @Elsevier GmbH, Urban \& Fischer Verlag Munchen. Volume 1, Edited by Putz, R. and Pabst, R. Lippincott Williams and Wilkins, Philadelphia 2001, Figures 66, 68, 82 and 83).

\section{Childhood to Adulthood Facial Remodeling}

As one ages from childhood to adulthood, there is a constant growth and remodeling of the facial bones, which results in changes in the facial morphology. Overall, there is forward and downward growth of the face with progressing age. As a generality, the farther structures lie from the neurocranium, the longer they grow and the more they increase in size. Thus, growth of the mandible begins later and continues longer than does the growth of the midface and orbits.

During the first decade of life, the forehead grows in an anterior and slightly upward direction. This contributes to the elevation and widening of the nasal bridge (Fig $11 B,-D)$.

The zygomatic/maxillary region grows progressively posteriorly as the dental arch becomes elongated by addition of new bone on the posterior margin of the maxilla. That part of the maxilla anterior to the zygomatic arch regresses while the posterior portion increases in size. ${ }^{6,7}$

The backward movement of the malar region and the forward growth of the supraorbital region serve to draw out the anteroposterior dimensions of the face. The inferior orbital rim and the superior orbital rim are in the same coronal plane in the young face, but in the older face, the supraorbital region protrudes forward of the cheek (Fig 12).

In the transverse plane, the maxillary bones and the ethmoid bodies grow apart from one another so that the interocular distance increases with age (Fig 12C). The movement of the nasal area combined with the malar movement results in an increase in the vertical size and the width of the upper part of each nasal cavity (Fig 12D). The mandibular ramus becomes progressively deeper in its anteroposterior dimension. The ramus also increases in vertical dimension, accommodating the marked downward growth of the nasomaxillary complex and the eruption of the teeth (Fig 12 $A,-C)$.

The young face appears somewhat brachycephalic because it is relatively wide and vertically short. The dentition has not yet become fully established, and the jaws have not yet grown to their full vertical extent. The young face also appears small compared with the cranium when the craniofacial sizes are compared with an adult skull (Fig 11). Thus, compared with an adult face, the young face appears "cute," with wide-set large eyes, a small jaw, a small "pug” nose, prominent cheeks, a high flat forehead 

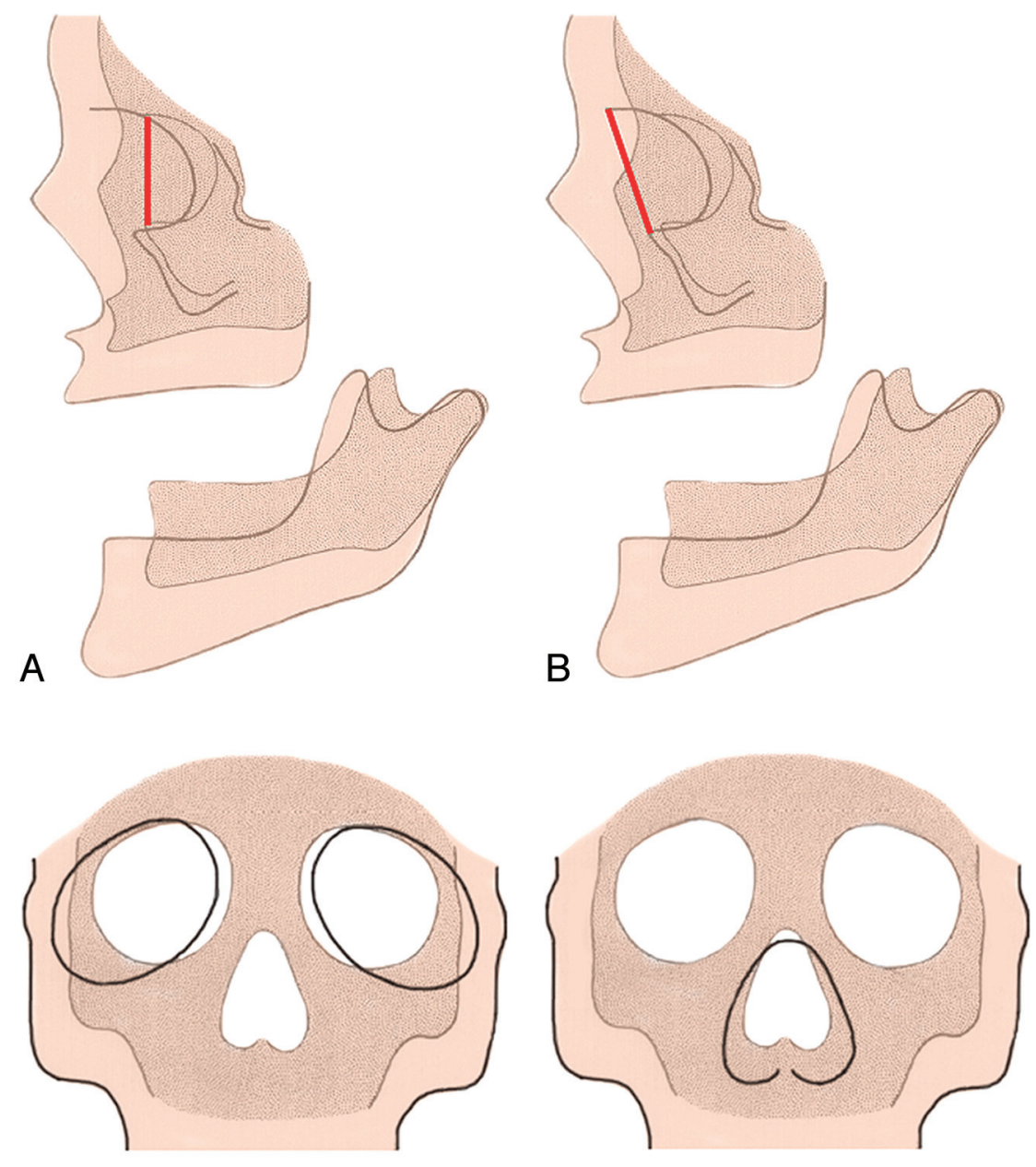

C

D

FIG 12. Lateral diagram of the fetal skull ( $A$ ) (darker areas) and the adult skull (B) (lighter areas) shows that the inferior orbital rim and the superior orbital rim are in the same plane in the young face (dark line in $A$ ), but in the older face, the supraorbital region protrudes forward of the cheek (dark line in $B$ ). Frontal diagrams ( $C$ and $D$ ) show that as the maxillary bones and the ethmoid bodies separate from one another, this movement increases the lateral growth of the interocular distance. As a result, the orbits enlarge and shift laterally (C). The movement of the nasal area combined with the malar movement results in an increase in the vertical size and the width of the upper part of each nasal cavity $(D)$. (Modified with permission from Enlow D. A Morphogenetic Analysis of Facial Growth. Am J Orthodontics 1966;52:283-299. Figures 1 and 4)

without full eyebrows ridges, a low nasal bridge, and a small mouth.

\section{REFERENCES}

1. Kim CH, Park HW, Kim K, et al. Early development of the nose in human embryos: a stereomicroscopic and histologic analysis. $\mathrm{La}$ ryngoscope 2004;114:1791-800

2. Warbrick JG. The early development of the nasal cavity and upper lip in the human embryo. J Anat 1960;94:351-62

3. Abrahams JJ, Poon CS, Hayt MW. Embryology and anatomy of the jaw and dentition. In: Som PM, Curtin HD, eds. Head and Neck Imaging. Philadelphia: Elsevier; 2011:1425-41

4. Gasser R. The development of the facial muscles in man. Am J Anat 1967;120:357-76

5. Scott JH. The growth in width of the facial skeleton. Am J Orthod 1957;43:366-71
6. Scott JH. The growth of the cranio-facial skeleton. Ir J Med Sci 1962;37:276-86

7. Enlow D. A Morphogenetic analysis of facial growth. Am J Orthod 1966;52:283-99

8. Levine HL, Clemente MP. Surgical anatomy of the paranasal sinus. In: Levine HL, Clemente MP, eds. Sinus Surgery: Endoscopic and Microscopic Approaches. New York: Thieme; 2005

9. Cochard LR, ed. Netter's Atlas of Human Embryology. Teterboro, New Jersey; Icon Learning Systems; 2002

10. Netter FL, Craig J, Machado C. netterimages.com. Netter Illustrations. Elsevier. www.netterimages.com. Accessed 2011

11. Human Embryology Organogenesis. 19.1. Face and upper foregut. http://embryology.ch/anglais/sdigestive/gesicht05.html. Accessed 2011

12. www.embryo.chronolab.com/teeth.html. Accessed 2011

13. Pultz HvR, Pabst R, eds. Sobotta: Atlas der Anatomie des Menschen. Philadelphia: Lippincott Williams and Wilkins; 2001 